\title{
Factors influencing sperm retrieval following testicular sperm extraction in nonobstructive azoospermia patients
}

\author{
Peyman Salehi ${ }^{*}$, Marzieh Derakhshan-Horeh ${ }^{2 *}$, Zakiye Nadeali ${ }^{3}$, Majid Hosseinzadeh ${ }^{3,4}$, Erfan Sadeghi, \\ Mohammad Hossein Izadpanahi ${ }^{7}$, Mansour Salehi ${ }^{3,8}$
}

${ }^{1}$ Shahid Beheshti Infertility Center, Isfahan University of Medical Sciences, Isfahan; ${ }^{2}$ Department of Anatomy, School of Medicine, Tehran University of Medical Sciences, Tehran; ${ }^{3}$ Medical Genetics Laboratory, Al-Zahra University Hospital, Isfahan; ${ }^{4}$ Department of Medical Genetics, School of Medicine, Tehran University of Medical Sciences, Tehran; ${ }^{5}$ Department of Biostatistics and Epidemiology, School of Public Health, Kermanshah University of Medical Sciences, Kermanshah; ${ }^{6}$ Department of Biostatistics and Epidemiology, School of Public Health, Isfahan University of Medical Sciences, Isfahan; ${ }^{7}$ Department of Urology, Isfahan University of Medical Sciences, Isfahan; ${ }^{8}$ Department of Genetics and Molecular Biology, Medical School, Isfahan University of Medical Sciences, Isfahan, Iran

Objective: Azoospermia owing to testicular disorders is the most severe manifestation of male infertility. The main concern for patients with nonobstructive azoospermia (NOA) is the probability of successful sperm retrieval following testicular sperm extraction (TESE). Therefore, the goal of this study was to determine predictive factors correlated with sperm retrieval.

Methods: We assessed the testicular histopathological patterns, the choice of TESE surgical procedure, hormone levels, and chromosomal abnormalities in patients with NOA $(n=170)$. The histopathology specimens were analyzed based on the histopathological patterns of hypospermatogenesis, maturation arrest, and Sertoli cell-only syndrome.

Results: The mean rate of sperm retrieval was $48.8 \%$. The rate of sperm retrieval was significantly higher in the hypospermatogenesis group than in the other groups $(p<0.001)$. There was a positive correlation between micro-TESE (vs. conventional TESE) and the sperm retrieval rate (odds ratio, $8.077 ; p<0.01$ ). A logistic regression model demonstrated that high levels of follicle-stimulating hormone $(\mathrm{FSH})$ and small testicular volume were significantly associated with lower chances of successful sperm retrieval.

Conclusion: Some parameters, including testicular histopathology patterns, FSH levels, testicular volume, and method of TESE surgery, may be able to predict the chances of obtaining spermatozoa in patients with NOA. However, despite the efficiency of some predictive models, the hope of retrieving any functioning spermatozoa may be sufficient to disregard predictive factors of the success of intracytoplasmic sperm injection in these patients.

Keywords: Azoospermia; Pathology; Sperm retrieval

Received: Nov 14, 2016 · Revised: Jan 5, 2017· Accepted: Feb 2, 2017

Co-corresponding authors: Marzieh Derakhshan-Horeh

Department of Anatomy, School of Medicine, Tehran University of Medical

Sciences, Tehran Province, Tehran, District 6, Pour Sina St, Iran

Tel: +98-216509432 Fax:+98-2166404377 E-mail:mh_derakhshan@yahoo.com

Peyman Salehi

Shahid Beheshti Infertility Center, Isfahan University of Medical Sciences, P.O. Box 319, Hezar-Jerib Ave., Isfahan 8174673461 , Iran

Tel: +98-313-2367001 Fax:+98-313-2367001 E-mail:dr_p_salehi@yahoo.com

* These authors contributed equally to this study.

* The study was supported by Shahid Beheshti Infertility Center, Isfahan University of Medical Sciences, Isfahan, Iran.

This is an Open Access article distributed under the terms of the Creative Commons Attribution Non-Commercial License (http://creativecommons.org/licenses/by-nc/4.0/) which permits unrestricted non-commercial use, distribution, and reproduction in any medium, provided the original work is properly cited.

\section{Introduction}

Azoospermia originating from testicular disorders, defined as the complete absence of spermatozoa in ejaculation, is the most severe manifestation of male infertility [1].

Sperm retrieval after testicular sperm extraction (TESE) for infertility treatment by intracytoplasmic sperm injection (ICSI) is the main challenge in patients with nonobstructive azoospermia (NOA). One of the effective parameters that should be considered in the management of patients with NOA undergoing ICSI treatment with TESE or micro-TESE procedures is the ability to predict the rate of spermatozoa recovery in these patients. Understanding these parameters is 
also important for counseling the patient and his partner [2].

The seminiferous tubules of patients with NOA display differing degrees of deficient spermatogenesis, including hypospermatogenesis, tubular sclerosis, Sertoli cell-only syndrome (SCOS), and maturation arrest (MA) [3-5]. In order to overcome the problem of failure to produce spermatozoa in ejaculation in patients with NOA, a TESE procedure is performed in order to find the focal area of spermatogenesis [6]. Evaluation based on histopathological patterns following a TESE operation may be effective in predicting successful sperm retrieval. Thus, it would be of great value in the management of patients with NOA [7-10].

Patients with NOA have an increased risk of chromosomal abnormalities [11]. Genetic alterations and an abnormal karyotype are considered to be prognostic factors affecting sperm retrieval. For example, a higher rate of sperm retrieval following TESE is reported in patients with Klinefelter syndrome [12].

In addition, other suggested predictors of a successful or unsuccessful TESE operation include the levels of serum follicle-stimulating hormone (FSH), luteinizing hormone (LH), inhibin, and testis size [13].

The present study aimed to identify valuable factors for predicting and managing TESE outcomes in patients with NOA. Based on our study, this information would be useful in counseling couples about the rate of successful sperm retrieval after an invasive surgical procedure such as TESE in azoospermic men, or even the chances of success for oocyte pick-up in their partners.

\section{Methods}

\section{Patient population}

A retrospective cohort study was performed at the Shahid Beheshti Hospital in Isfahan, Iran. A total of 170 patients with NOA who were referred for fertility treatment from 2013 to 2016 were included in this study. Informed consent was obtained from all patients before inclusion in the study. All patients were confirmed to have NOA using at least two semen analyses according to World Health Organization guidelines [14], without any sperm and with normal $\mathrm{pH}$ and volume ( $>1.5 \mathrm{~mL}$ ), indicating both functional seminal vesicles and open ejaculatory ducts. Additionally, the assessment of diagnostic parameters, including clinical history, physical examination, hormonal analysis, and pathology assay should be performed to exclude obstructive azoospermia.

For the hormonal assay, serum FSH, LH, testosterone, and prolactin concentrations were measured by an electrochemical luminescence assay in the morning. The reference ranges were considered to be 1.5 to $12.45 \mathrm{mlU} / \mathrm{mL}$ for $\mathrm{FSH}, 1.7$ to $8.6 \mathrm{mlU} / \mathrm{mL}$ for $\mathrm{LH}, 2.84$ to $8 \mathrm{ng} / \mathrm{mL}$ for testosterone, and 1.8 to $20.3 \mathrm{ng} / \mathrm{mL}$ for prolactin. Testicular volume was measured by physical examination using an orchidometer.
A volume of $<15 \mathrm{~mL}$ per testis was considered to indicate small testicular size. The mean body mass index of the patients was $25.2 \pm 3.9$ $\mathrm{kg} / \mathrm{m}^{2}$ (range, $16-42 \mathrm{~kg} / \mathrm{m}^{2}$ ) and the couples had a median duration of infertility of 3.5 years.

\section{TESE procedure}

The TESE and micro-TESE procedures were performed according to the standard protocols described by Schlegel for micro-TESE [15]. Briefly, for both surgical procedures, a median raphe incision was made in the scrotum, the tunica vaginalis was opened, and the testis was delivered. In TESE procedures, a longitudinal testicular biopsy was obtained according to the size of the testis (at least $10 \mathrm{~mm} \times 5$ $\mathrm{mm}$ ). In micro-TESE procedures, using an operating microscope with $\times 25$ to $\times 40$ magnification, enlarged and opaque seminiferous tubules were removed. Then, the sperm recovery procedure was carried out based on the standard protocol by the embryologist. If any viable sperm were present in a wet preparation, they were used or cryopreserved for future ICSI treatment. Furthermore, the histopathology specimen was analyzed. Based on the most predominant histopathological patterns, patients with NOA were classified according to testicular histology as being affected by hypospermatogenesis, MA, or SCOS. In this study, the MA group is defined by either complete or partial MA. The hypospermatogenesis group includes mild to severe hypospermatogenesis. The presence of one or more spermatozoon in the testicular extraction was considered as successful sperm retrieval or a successful TESE outcome.

\section{Cytogenetic analysis}

Karyotyping was performed according to standard methods on phytohemagglutinin-stimulated peripheral lymphocyte cultures [16]. Blood cells were cultured at Roswell Park Memorial Institute 1640 medium, including $10 \%$ fetal bovine serum and $2 \%$ phytohemagglutinin at $37^{\circ} \mathrm{C}$. After 68 to 72 hours, Colcemid (Sigma-Aldrich, St.

Table 1. Statistical distribution of predictive factors according to the type of testicular histopathology

\begin{tabular}{lccr}
\hline Factor & MA & SCOS & \multicolumn{1}{c}{$\begin{array}{c}\text { Hyposper- } \\
\text { matogenesis }\end{array}$} \\
\hline FSH & $18.27 \pm 10.26$ & $22.59 \pm 10.99$ & $9.73 \pm 7.98$ \\
LH & $8.26 \pm 6.55$ & $10.11 \pm 5.66$ & $7.76 \pm 7.00$ \\
Prolactin & $9.22 \pm 5.06$ & $11.14 \pm 4.98$ & $10.34 \pm 8.19$ \\
Testosterone & $3.54 \pm 2.14$ & $4.89 \pm 2.79$ & $4.48 \pm 2.98$ \\
Age & $34.86 \pm 5.71$ & $31.33 \pm 5.24$ & $32.41 \pm 5.18$ \\
Chromosome abnormality & $6(13.0)$ & $10(13.5)$ & $6(12.0)$ \\
Sperm retrieval & $20(43.5)$ & $16(21.6)$ & $47(94.0)$
\end{tabular}

Values are presented as mean \pm standard deviation or number (\%). MA, maturation arrest; SCOS, Sertoli cell-only syndrome; FSH, follicle-stimulating hormone; $\mathrm{LH}$, luteinizing hormone. 
Louis, MO, USA) was added to the cell. Then, the cultures were harvested and cells in metaphase were stained with trypsin $\mathrm{G}$ banding. Chromosomal abnormalities were designated using the standard International System for Human Cytogenetic Nomenclature guidelines [17].

\section{Statistical analysis}

Summary measures for the factors, according to sperm retrieval and pathology, are presented in Tables 1 and 2, respectively. Binary and multinomial logistic regression models were evaluated for sperm retrieval and pathology as the response variables, respectively. For each model, independent variables and the estimated coefficients, odds ratios (ORs), and corresponding confidence intervals are given in Tables 2 and 3.
All analyses were carried out with SPSS ver. 16.0 (SPSS Inc., Chicago, IL, USA) and the significance level was considered to be $p<0.05$.

\section{Results}

\section{Histopathological evaluation of TESE in patients with NOA}

The mean age of all patients was $32.56 \pm 5.53$ years. Based on the histopathological findings, the 170 patients were classified into three groups: 74 (43.5\%) cases of SCOS, 46 (27.1\%) cases of MA, and 50 (29.4\%) cases of hypospermatogenesis. The levels of FSH were significantly in excess of the normal ranges in the SCOS and MA groups when compared to the hypospermatogenesis group (MA: OR, 1.146, $p=0.034$; SCOS: OR, 1.190, $p=0.007$ ). In addition, prolactin levels were significantly higher in the SCOS group than in the hyposper-

Table 2. Multinomial logistic regression model with pathology as the nominal response variable

\begin{tabular}{|c|c|c|c|c|c|c|}
\hline \multirow{2}{*}{ Pathology } & \multirow{2}{*}{ Factor } & \multirow{2}{*}{$\beta$} & \multirow{2}{*}{ SE } & \multirow{2}{*}{ OR } & \multicolumn{2}{|c|}{$95 \%$ Cl for OR } \\
\hline & & & & & Lower bound & Upper bound \\
\hline \multirow[t]{7}{*}{ MA } & FSH & 0.136 & 0.064 & $1.146^{*}$ & 1.010 & 1.300 \\
\hline & $\mathrm{LH}$ & -0.102 & 0.068 & 0.903 & 0.790 & 1.032 \\
\hline & Prolactin & 0.090 & 0.072 & 1.094 & 0.949 & 1.260 \\
\hline & Testosterone & -0.168 & 0.130 & 0.845 & 0.655 & 1.091 \\
\hline & Age & 0.068 & 0.059 & 1.070 & 0.954 & 1.201 \\
\hline & Normal karyotyping & 0.198 & 1.160 & 1.219 & 0.126 & 11.836 \\
\hline & No sperm retrieval & 2.815 & 0.912 & $16.696^{* *}$ & 2.793 & 99.823 \\
\hline \multirow[t]{7}{*}{ scos } & FSH & 0.174 & 0.065 & $1.190^{* *}$ & 1.048 & 1.352 \\
\hline & $\mathrm{LH}$ & -0.122 & 0.073 & 0.885 & 0.768 & 1.020 \\
\hline & Prolactin & 0.212 & 0.076 & $1.236^{* *}$ & 1.064 & 1.436 \\
\hline & Testosterone & -0.017 & 0.125 & 0.983 & 0.769 & 1.257 \\
\hline & Age & 0.008 & 0.063 & 1.008 & 0.891 & 1.140 \\
\hline & Normal karyotyping & -0.209 & 1.310 & 0.811 & 0.062 & 10.577 \\
\hline & No sperm retrieval & 3.833 & 0.943 & $46.189^{* * *}$ & 7.280 & 293.048 \\
\hline
\end{tabular}

$\mathrm{SE}$, standard error; $\mathrm{OR}$, odds ratio; $\mathrm{Cl}$, confidence interval; $\mathrm{MA}$, maturation arrest; $\mathrm{FSH}$, follicle-stimulating hormone; LH, luteinizing hormone; SCOS, Sertoli cellonly syndrome.

a) The reference category was hypospermatogenesis.

Significant at ${ }^{*} p<0.05 ;{ }^{* *} p<0.01 ;{ }^{* * *} p<0.001$.

Table 3. Multiple logistic regression model with sperm retrieval as the response variable

\begin{tabular}{|c|c|c|c|c|c|}
\hline \multirow{2}{*}{ Factor } & \multirow{2}{*}{$\beta$} & \multirow{2}{*}{ SE } & \multirow{2}{*}{ OR } & \multicolumn{2}{|c|}{$95 \% \mathrm{Cl}$ for OR } \\
\hline & & & & Lower bound & Upper bound \\
\hline FSH & -0.333 & 0.079 & $0.717^{* * * *}$ & 0.614 & 0.837 \\
\hline LH & 0.128 & 0.077 & 1.137 & 0.978 & 1.322 \\
\hline Prolactin & -0.111 & 0.067 & 0.895 & 0.784 & 1.021 \\
\hline Testosterone & 0.016 & 0.121 & 1.017 & 0.802 & 1.289 \\
\hline Age & 0.042 & 0.063 & 1.043 & 0.922 & 1.181 \\
\hline Chromosome abnormality & -0.083 & 0.978 & 0.921 & 0.135 & 6.262 \\
\hline Abnormal testis size & 2.358 & 0.818 & $10.565^{* *}$ & 2.127 & 52.475 \\
\hline Micro-TESE surgery & 2.089 & 0.675 & $8.077^{* *}$ & 2.153 & 30.305 \\
\hline
\end{tabular}

$\mathrm{SE}$, standard error; $\mathrm{OR}$, odds ratio; $\mathrm{Cl}$, confidence interval; $\mathrm{FSH}$, follicle-stimulating hormone; $\mathrm{LH}$, luteinizing hormone; TESE, testicular sperm extraction. Significant at ${ }^{* *} p<0.01 ;{ }^{* * *} p<0.001$. 
matogenesis group (OR, 1.236; $p=0.006)$, as shown in Table 2 . No associations were observed among age, chromosomal abnormalities, and histopathological patterns ( $p>0.05$ ) (Table 2). The rate of chromosomal abnormalities was $12.9 \%$ in all patients. The greatest number of patients with chromosomal abnormalities (17 cases) showed 47,XXY karyotyping. Other chromosomal abnormalities included $47, X Y Y$ (two cases), 46,XX male (one case), 46X,+mar (one case) and 46,XY,DelY q11.2 (one case).

\section{Sperm retrieval after TESE in patients with NOA}

Our results showed that the overall mean rate of sperm retrieval was $48.8 \%$. These rates were $21.6 \%$ in the SCOS group, $43.5 \%$ in the MA group, and $94 \%$ in the hypospermatogenesis group. Based on the multinomial logistic regression model, the rates of unsuccessful sperm retrieval were significantly higher in the MA and SCOS groups than in the hypospermatogenesis group (Table 2). The logistic regression model confirmed that high levels of FSH and small testicular volume were significantly associated with lower chances of successful sperm retrieval (OR, 0.717, $p<0.001 ; \mathrm{OR}, 10.5, p<0.01$, respectively) (Table 3). However, age, testosterone, and prolactin levels were not associated with positive sperm retrieval ( $p>0.05$ ). The results exhibited statistically significant differences in sperm retrieval between conventional TESE and micro-TESE procedures, and there was an eight-fold greater chance of sperm retrieval with the microTESE method than with conventional TESE. The association between chromosomal abnormalities and the rate of sperm retrieval was assessed. No associations were observed among the aforementioned factors and sperm retrieval $(p>0.05)$.

\section{Discussion}

In this study, we assessed the effects of the histopathological pattern, age, the choice of TESE surgical procedure (conventional TESE or micro-TESE), hormones, testis volume, and karyotyping on sperm retrieval in patients with NOA.

In infertility consultations for patients with NOA, it is essential to provide information on the chances of sperm being retrieved. $A$ failed TESE procedure on ovum pick-up day can have irreparable emotional effects and financial implications for both members of the couple [8]. Therefore, the evaluation of successful sperm retrieval based on genetic factors, hormones, and the histopathological status of patients may be useful in the management of patients with NOA.

Consistent with previous studies, in our study, SCOS was the most common histopathological pattern diagnosed (43.5\% of patients with NOA) $[13,18]$.

An elevated level of FSH was observed in all three histopathological groups. This increase in the observed concentrations of the hormone was related to the spermatogenetic defect and decrease in total number of testicular germ cells in patients with NOA. In accordance with the finding of Aydin et al. [18] of a significantly higher level of FSH and LH in patients with SCOS, our results showed that the level of FSH was significantly higher in the MA and, in particular, the SCOS groups than in the hypospermatogenesis group.

In our study, the mean rate of sperm retrieval was $48.8 \%$, and this rate was significantly higher in the hypospermatogenesis group than in the SCOS and MA groups ( $94 \%$ vs. $21.6 \%$ and $43.5 \%$, respectively). In accordance with earlier studies [19-21], the use of histopathological patterns could be considered as a valuable predictor for sperm recovery. Accordingly, the chance of sperm retrieval is higher in patients with hypospermatogenesis compared with patients with MA and SCOS. The efficacy of testicular histopathology on the results of ICSI after TESE has been assessed, but testicular histopathology does not positively affect the ICSI outcome [13]. However, Aydin et al. [18] demonstrated that once successful sperm retrieval was achieved, fertilization and clinical pregnancy rates were similar in patients with NOA. In addition, Hessel et al. [6] reported that a simple cytological evaluation of the TESE specimen by defining the ratios among Sertoli cells, pachytene spermatocytes, and spermatozoa could be a valuable predictor for the outcome of fertility treatment in patients with NOA.

Based on our findings, high levels of FSH and small testicular volume were significantly associated with lower chances of successful sperm retrieval. These factors appear to be useful for predicting the success of TESE. These findings may be in part consistent with the study of Cissen et al. [22], which presented a model for the prediction of successful TESE. They showed that lower levels of FSH and $\mathrm{LH}$, higher male age, higher levels of serum testosterone, and diagnosis of idiopathic NOA or an azoospermia factor-c deletion were able to predict the success of sperm retrieval.

Our results showed better outcomes (higher rates of sperm retrieval) from the micro-TESE procedure than from conventional TESE. Currently, there is a trend toward using micro-TESE procedures due to the low complication rates, better approach to the problem of focal spermatogenesis observed in patients with NOA, and higher chance of sperm retrieval, particularly for patients with the histological pattern of SCOS [23]. In some clinics, micro-TESE is performed on the day of oocyte retrieval using fresh sperm for ICSI. However, in our clinic, to ensure the availability of sperm before beginning the ICSI cycle, a diagnostic biopsy was carried out and then the obtained sperm was frozen. A second TESE procedure may be necessary on the day of oocyte pick-up, if there is not enough thawed sperm.

Although no association was observed between chromosomal abnormalities and the histopathological pattern or sperm retrieval, it seems that the detection of chromosome abnormalities aided the 
assessment of the potential rate of sperm retrieval in patients with NOA. However, in this research, evaluation of chromosomal abnormalities was performed by karyotype analysis, which detects largescale genetic changes and does not detect submicroscopic alterations in the DNA sequence. Therefore, following the detection of a normal karyotype, it would be wise to perform complementary genetic tests in order to assess the critical genes involved in the process of spermatogenesis. The other reason for our observations may be related to the small number of patients.

Thus far, three studies have assessed the predictive factors of achieving pregnancy after TESE [24-26]. Although predictive models for sperm retrieval or pregnancy outcome after TESE have been designed to counsel patients with NOA about their chances of successfully obtaining spermatozoa, they have not generally been implemented in clinical practice [27].

In conclusion, valuable factors for predicting successful sperm retrieval during TESE, including testicular histopathology patterns, FSH levels, testicular volume, and the use of micro-TESE, were examined in our study, allowing an estimation of the chances of obtaining spermatozoa in patients with NOA. Accordingly, the combination and simultaneous interpretation of the factors studied here, such as histopathological patterns, hormone levels, and karyotyping, help provide an accurate diagnosis of testicular failure and subsequently help the clinician to pursue the appropriate methods of treatment for these patients. In addition, it should be considered that the hope of retrieving any spermatozoa at all might be a sufficient cause for attempting ICSI in these patients, regardless of the predictive factors.

\section{Conflict of interest}

No potential conflict of interest relevant to this article was reported.

\section{References}

1. Esteves SC, Lee W, Benjamin DJ, Seol B, Verza S Jr, Agarwal A. Reproductive potential of men with obstructive azoospermia undergoing percutaneous sperm retrieval and intracytoplasmic sperm injection according to the cause of obstruction. J Urol 2013;189:232-7.

2. Vicari E, Grazioso C, Burrello N, Cannizzaro M, D'Agata R, Calogero AE. Epididymal and testicular sperm retrieval in azoospermic patients and the outcome of intracytoplasmic sperm injection in relation to the etiology of azoospermia. Fertil Steril 2001;75:2156.

3. Levin HS. Testicular biopsy in the study of male infertility: its current usefulness, histologic techniques, and prospects for the future. Hum Pathol 1979;10:569-84.
4. Lellei I, Magyar E, Erdei E. Histological evaluation of multiple testicular biopsies helping assisted reproduction. Pathol Res Pract 2001;197:727-33.

5. McLachlan RI, Rajpert-De Meyts E, Hoei-Hansen CE, de Kretser DM, Skakkebaek NE. Histological evaluation of the human testis-approaches to optimizing the clinical value of the assessment mini review. Hum Reprod 2007;22:2-16.

6. Hessel M, de Vries M, D'Hauwers KW, Fleischer K, Hulsbergen-van de Kaa CA, Braat DD, et al. Cytological evaluation of spermatogenesis: a novel and simple diagnostic method to assess spermatogenesis in non-obstructive azoospermia using testicular sperm extraction specimens. Andrology 2015;3:481-90.

7. Jezek D, Knuth UA, Schulze W. Successful testicular sperm extraction (TESE) in spite of high serum follicle stimulating hormone and azoospermia: correlation between testicular morphology, TESE results, semen analysis and serum hormone values in 103 infertile men. Hum Reprod 1998;13:1230-4.

8. Ezeh UI, Taub NA, Moore HD, Cooke ID. Establishment of predictive variables associated with testicular sperm retrieval in men with non-obstructive azoospermia. Hum Reprod 1999;14:100512.

9. Tournaye H, Verheyen G, Nagy P, Ubaldi F, Goossens A, Silber S, et al. Are there any predictive factors for successful testicular sperm recovery in azoospermic patients? Hum Reprod 1997;12:80-6.

10. Sousa M, Cremades N, Silva J, Oliveira C, Ferraz L, Teixeira da Silva $\mathrm{J}$, et al. Predictive value of testicular histology in secretory azoospermic subgroups and clinical outcome after microinjection of fresh and frozen-thawed sperm and spermatids. Hum Reprod 2002;17:1800-10.

11. Pylyp LY, Spinenko LO, Verhoglyad NV, Zukin VD. Chromosomal abnormalities in patients with oligozoospermia and non-obstructive azoospermia. J Assist Reprod Genet 2013;30:729-32.

12. Schiff JD, Palermo GD, Veeck LL, Goldstein M, Rosenwaks Z, Schlegel PN. Success of testicular sperm extraction [corrected] and intracytoplasmic sperm injection in men with Klinefelter syndrome. J Clin Endocrinol Metab 2005;90:6263-7.

13. Abdel Raheem A, Garaffa G, Rushwan N, De Luca F, Zacharakis E, Abdel Raheem T, et al. Testicular histopathology as a predictor of a positive sperm retrieval in men with non-obstructive azoospermia. BJU Int 2013;111:492-9.

14. Cooper TG, Noonan E, von Eckardstein S, Auger J, Baker HW, Behre HM, et al. World Health Organization reference values for human semen characteristics. Hum Reprod Update 2010;16: 231-45.

15. Dabaja AA, Schlegel PN. Microdissection testicular sperm extraction: an update. Asian J Androl 2013;15:35-9.

16. Barch MJ, Knutsen T, Spurbeck JL. The AGT cytogenetics labora- 
tory manual. Philadelphia: Lippincott-Raven; 1997.

17. Shaffer LG, Slovak ML, Campbell LJ. An International System for Human Cytogenetic Nomenclature (2009): recommendations of the International Standing Committee on human cytogenetic nomenclature. Basel: Karger; 2009.

18. Aydin T, Sofikerim M, Yucel B, Karadag M, Tokat F. Effects of testicular histopathology on sperm retrieval rates and ICSI results in non-obstructive azoospermia. J Obstet Gynaecol 2015;35:82931.

19. Weedin JW, Bennett RC, Fenig DM, Lamb DJ, Lipshultz LI. Early versus late maturation arrest: reproductive outcomes of testicular failure. J Urol 2011;186:621-6.

20. Seo JT, Ko WJ. Predictive factors of successful testicular sperm recovery in non-obstructive azoospermia patients. Int J Androl 2001;24:306-10.

21. Yang J, Liu JH, Zou XF, Yuan YH, Xiao RH, Wu GQ, et al. Sperm retrieval and the predictive parameter of non-obstructive azoospermia: a meta-analysis of literatures 1990 to 2008. Zhonghua Yi Xue Za Zhi 2008;88:2131-5.

22. Cissen M, Meijerink AM, D'Hauwers KW, Meissner A, van der Weide $\mathrm{N}$, Mochtar $\mathrm{MH}$, et al. Prediction model for obtaining spermatozoa with testicular sperm extraction in men with nonobstructive azoospermia. Hum Reprod 2016;31:1934-41.

23. Deruyver Y, Vanderschueren D, Van der Aa F. Outcome of microdissection TESE compared with conventional TESE in non-obstructive azoospermia: a systematic review. Andrology 2014;2: 20-4.

24. Meijerink AM, Cissen M, Mochtar MH, Fleischer K, Thoonen I, de Melker AA, et al. Prediction model for live birth in ICSI using testicular extracted sperm. Hum Reprod 2016;31:1942-51.

25. Silber SJ, Nagy Z, Devroey P, Camus M, Van Steirteghem AC. The effect of female age and ovarian reserve on pregnancy rate in male infertility: treatment of azoospermia with sperm retrieval and intracytoplasmic sperm injection. Hum Reprod 1997;12: 2693-700.

26. Vernaeve V, Staessen C, Verheyen G, Van Steirteghem A, Devroey $\mathrm{P}$, Tournaye $\mathrm{H}$. Can biological or clinical parameters predict testicular sperm recovery in 47,XXY Klinefelter's syndrome patients? Hum Reprod 2004;19:1135-9.

27. McLernon DJ, Bhattacharya S. Clinical decision-making in azoospermic men: in search of the ideal prediction model. Hum Reprod 2016;31:1931-3. 